\title{
AGN and starburst galaxies at low radio flux densities
}

S. K. Sirothia

NCRA-TIFR, India

E-mail: sirothia@ncra.tifr.res.in

D. J. Saikia

NCRA-TIFR, India

E-mail: djsencra.tifr.res.in

\section{Dennefeld}

Institut d'Astrophysique de Paris, 98bis Boulevard Arago, F-75014, Paris, France

E-mail: dennefeleiap.fr

\section{Burgarella}

Observatoire Astronomique Marseille Provence, Laboratoire d'Astrophysique de Marseille,

France

E-mail: denis.burgarelladoamp.fr

Deep surveys of the sky at low radio frequencies provide important inputs towards identifying AGN and starburst galaxies from both the structure and spectra of these sources. Such observations could also provide evidence of relic emission from earlier cycles of activity in an AGN, possibly caused by periodic 'feeding' of a supermassive black hole. In this paper we present deep radio observations at low radio frequencies of selected fields using the Giant Metrewave Radio Telescope (GMRT). We identify possible tailed radio sources in clusters of galaxies at moderate redshifts, summarise our results on the flattening of the source counts which we have seen for the first time at these wavelengths and which is likely to be due to a combination of both starburst galaxies and low-luminosity AGN. We present images of selected sources, some of which have very steep spectra, and discuss the nature of these objects.

25th Texas Symposium on Relativistic Astrophysics

December 6-10, 2010

Heidelberg, Germany 


\section{Introduction}

Multiwavelength studies are essential to understand the properties, formation and evolution of galaxies over cosmic time [1, 2]. At radio wavelengths, we are able to probe deep into the central regions of active galaxies without being affected by the dust and gas, which has helped to distinguish between starburst galaxies and those that host active galactic nuclei (AGN). The radio sources with a flux density greater than $\sim 1 \mathrm{mJy}$ at $1400 \mathrm{MHz}$ are almost entirely due to an AGN which is powered by accretion onto a SMBH. However, below $\sim 1$ mJy the radio sources are possibly due a combination of starburst galaxies and a population of low-luminosity AGN [3, 4, 5, 6]. The star formation in these galaxies could be triggered by interactions and mergers, and thereby contain an imprint of the evolution of these galaxies over cosmic time.

The radio structures of the extended sources also help probe their environments. For example tailed radio sources, such as the wide-angle tailed (WAT) sources, can, in principle, be used to probe clusters and groups of galaxies at moderate and high redshifts [7]. Low-frequency radio observations help determine their spectral indices over a large frequency range and help identify very steep-spectrum sources, with spectral indices $\alpha>1.3\left(\mathrm{~S} \propto v^{-\alpha}\right)$, which could be either highredshift galaxies [\$] or relic sources [9]. These observations are critical for studying episodic activity in AGN [10, 11], and provide valuable information towards understanding the evolution of galaxies over cosmic time.

\section{GMRT observations of selected fields}

To explore some of the issues outlined earlier, we have been making deep observations of several selected fi elds at low radio frequencies using the Giant Metrewave Radio Telescope (GMRT). We describe the results from two of the fi elds.

The ELAIS-N1 (European Large Area Infrared Space Observatory Survey) fi eld has been chosen in a region of the sky with low-IR foreground emission, to allow detection of fainter and possibly more distant galaxies. GMRT observations of the ELAIS-N1 fi eld at $325 \mathrm{MHz}$ has an rms noise fi gure of $\sim 40 \mu \mathrm{Jy}_{\text {beam }}^{-1}$ towards the centre of the fi eld, and is amongst the deepest images made at this frequency [12]. The GMRT image of an area of $30 \times 30 \mathrm{arcmin}^{2}$ from our observations at $325 \mathrm{MHz}$, and for comparison, the corresponding images of the same area at $610 \mathrm{MHz}$ with the GMRT from Garn et al. [13] and the Faint Images of the Radio Sky at Twenty-centimetres (FIRST) and NRAO VLA Sky Survey (NVSS) at $1400 \mathrm{MHz}$ are shown in Figure 1. Although the brighter sources are seen clearly in all the images, many more sources are seen in the $325-\mathrm{MHz}$ image due to the deeper surface brightness sensitivity of this image.

The Extended Chandra Deep Field-South (ECDFS) is one of the most extensively studied regions of the sky. The GMRT observations have a median rms noise in the central region of 2.5 mJy beam ${ }^{-1}$ at $153 \mathrm{MHz}$ and $75 \mu \mathrm{Jy}_{\text {beam }}{ }^{-1}$ at $325 \mathrm{MHz}$. The images with angular resolutions of $25.2 \times 14.5 \operatorname{arcsec}^{2}$ along a position angle (PA) of $24^{\circ}$, and $10.8 \times 7.3 \operatorname{arcsec}^{2}$ along a PA of $16^{\circ}$ are shown in Figure 8, along with a VLA image at $1400 \mathrm{MHz}$ from Miller et al. [14].

To illustrate the range of structures, we show the GMRT images of three wide-angle tailed sources which appear to be at moderate redshifts (Figure 2), and four of the galaxies from the ECDFS fi eld, (Figure 4 ) along with their VLA images at $1400 \mathrm{MHz}$. These sources have spec- 

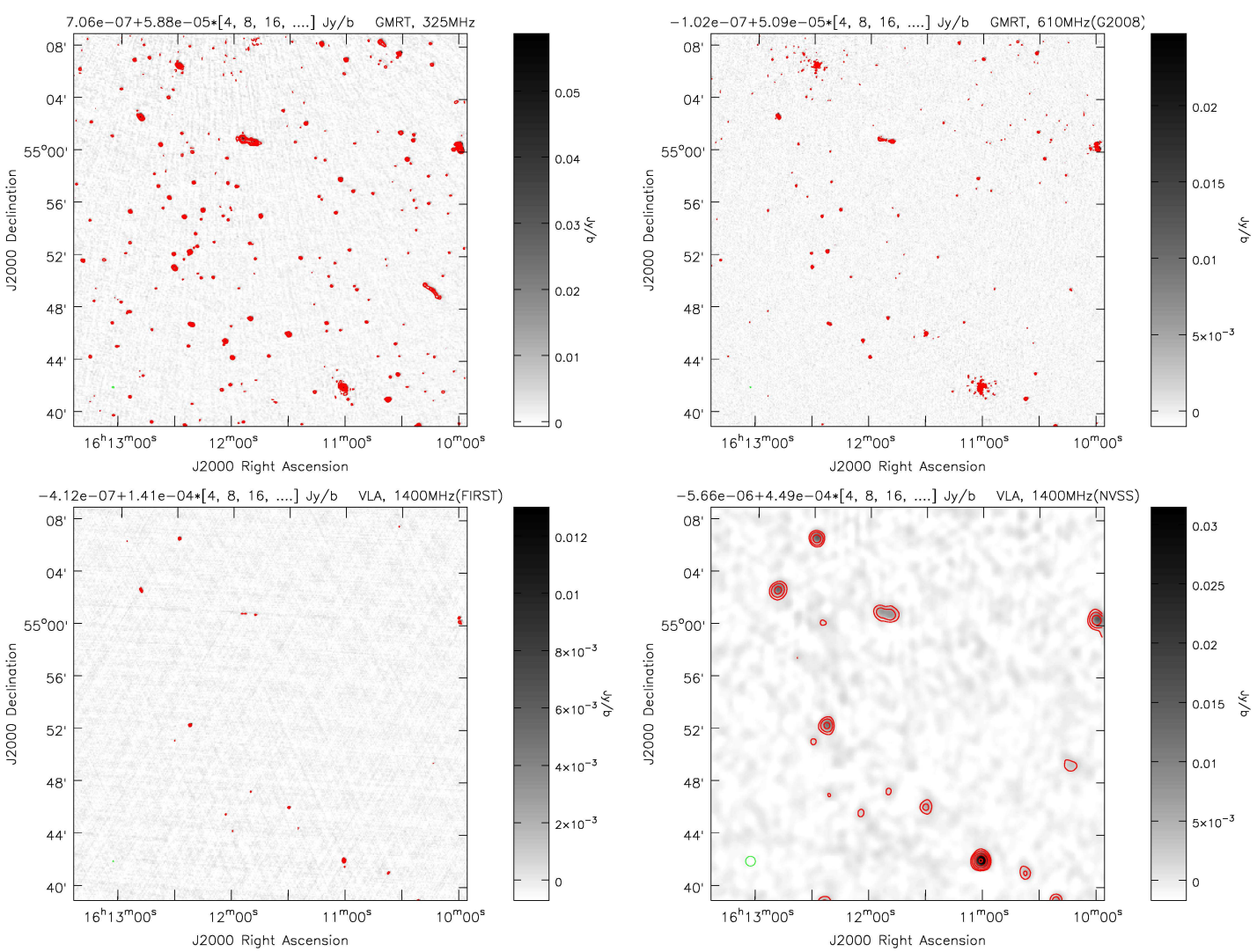

Figure 1: GMRT image of an area of $30 \times 30 \operatorname{arcmin}^{2}$ from our observations at $325 \mathrm{MHz}$ (top left), and the corresponding images of the same area at $610 \mathrm{MHz}$ with the GMRT [13] (top right), and from FIRST (bottom left) and NVSS (bottom right) at $1400 \mathrm{MHz}$, reproduced from [12]. In this figure and in all the images presented here the contour levels in units of $\mathrm{Jy}_{\text {beam }}{ }^{-1}$ are represented by mean $+\mathrm{rms} \times(\mathrm{n})$ where $\mathrm{n}$ is the multiplication factor. These levels are shown above each image. All negative contours appear as dashed lines.
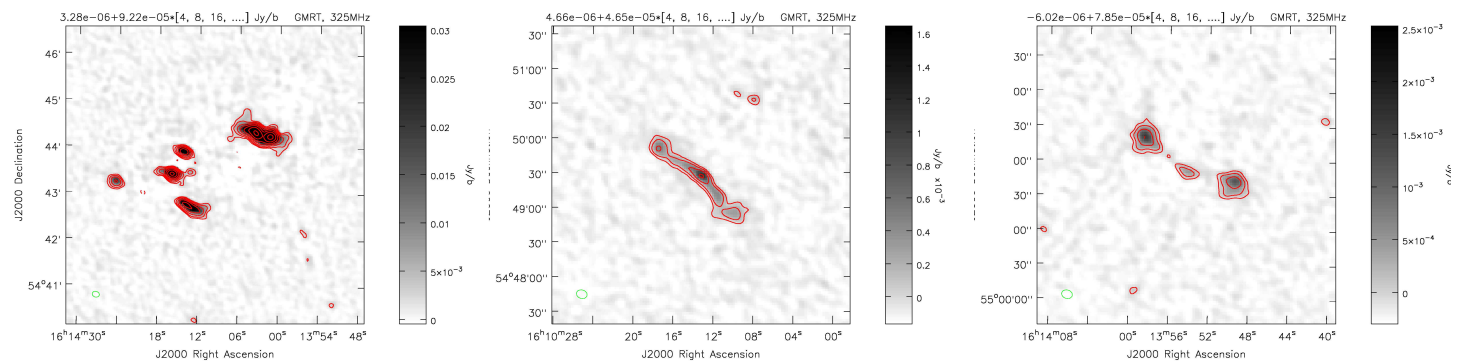

Figure 2: GMRT images at $325 \mathrm{MHz}$ of possible wide-angle tailed sources in the ELAIS-N1 field. 

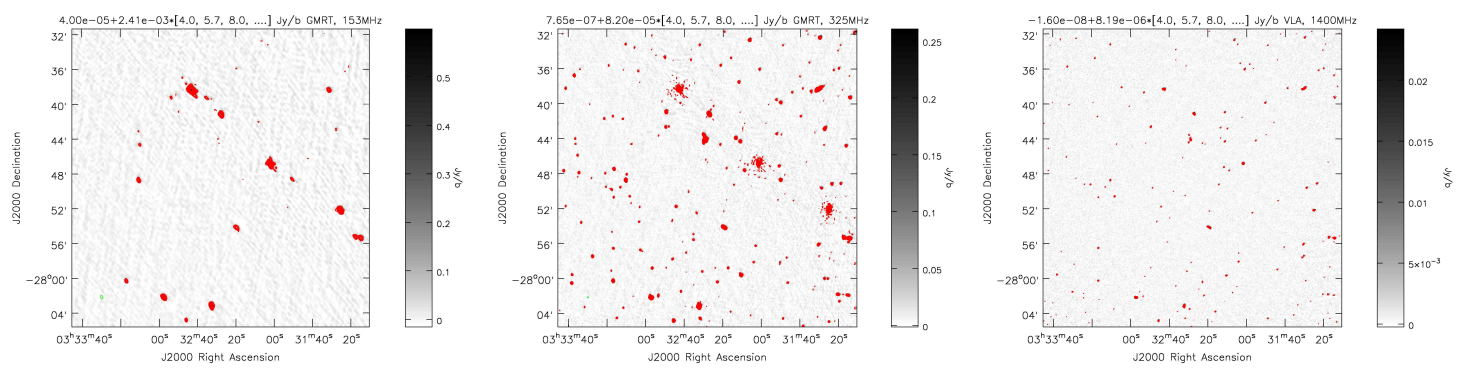

Figure 3: GMRT images at $153 \mathrm{MHz}$ (left) and 325-MHz (middle) of the ECDFS field, along with the VLA 1400-MHz image (right) made from the data release of Miller et al. [14.

troscopic redshifts available. While J033228-274355 appears to be an asymmetric double-lobed source with a jet-like structure towards the north, J033237-275004 is a single compact source at a redshift of 3.7638. J033242-273817 is at a redshift of 2.7301 and is the most luminous source known in our sample, while J033219-275408 is at a redshift of 0.9640. Although three of the four images shown here have a double-lobed structure, most of the sources in our sample are single sources.

\section{Spectral indices}

We have estimated the spectral indices of the sources detected in the low-frequency observations of both the ELAIS-N1 and ECDFS fi elds. For ELAIS-N1, the spectral indices for the sources which are within $0.7^{\circ}$ of the phase centre, the HPBW at $325 \mathrm{MHz}$, and which also appear either in the catalogues of Garn et al. or FIRST have been estimated. For further details see Sirothia et al. [12]. The median value of $\alpha_{325}^{1400}$ for sources in the ELAIS-N1 fi eld is 0.83 , with four candidate Giga-Hertz Peaked Spectrum (GPS) sources and 30 very steep spectrum objects with $\alpha>1.3$. In the ECDFS fi eld we identify a further 19 sources with a very steep spectrum.

We have investigated possible dependence of spectral index on flux density. The plot of $\alpha_{325}^{1400}$ vs flux density at $325 \mathrm{MHz}$ for the ELAIS-N1 fi eld is shown in Figure $\mathrm{G}$. Since a large fraction of sources in this fi eld have limits to their spectral indices, it is diffi cult to determine any dependence of spectral index on flux density. A similar plot for the ECDFS fi eld shown in Figure $\$$, shows that most of the sources have $\alpha>0.5$, with a median value of 0.95 , and that there is a larger scatter in the values of spectral indices for sources below a mJy. This is possibly due to a combination of both different populations of sources at low flux densities and larger errors in the spectral indices at low flux densities. The fi gure does not show a signifi cant dependence of spectral index on flux density for these sources.

\section{Source counts}

The source counts at $325 \mathrm{MHz}$ for the ELAIS-N1 fi eld has been reported earlier by Sirothia et al. [12]. The normalized differential source counts showed a flattening at about a mJy, which had been seen earlier in only higher-frequency studies. In Figure 6, we show the normalized differential 

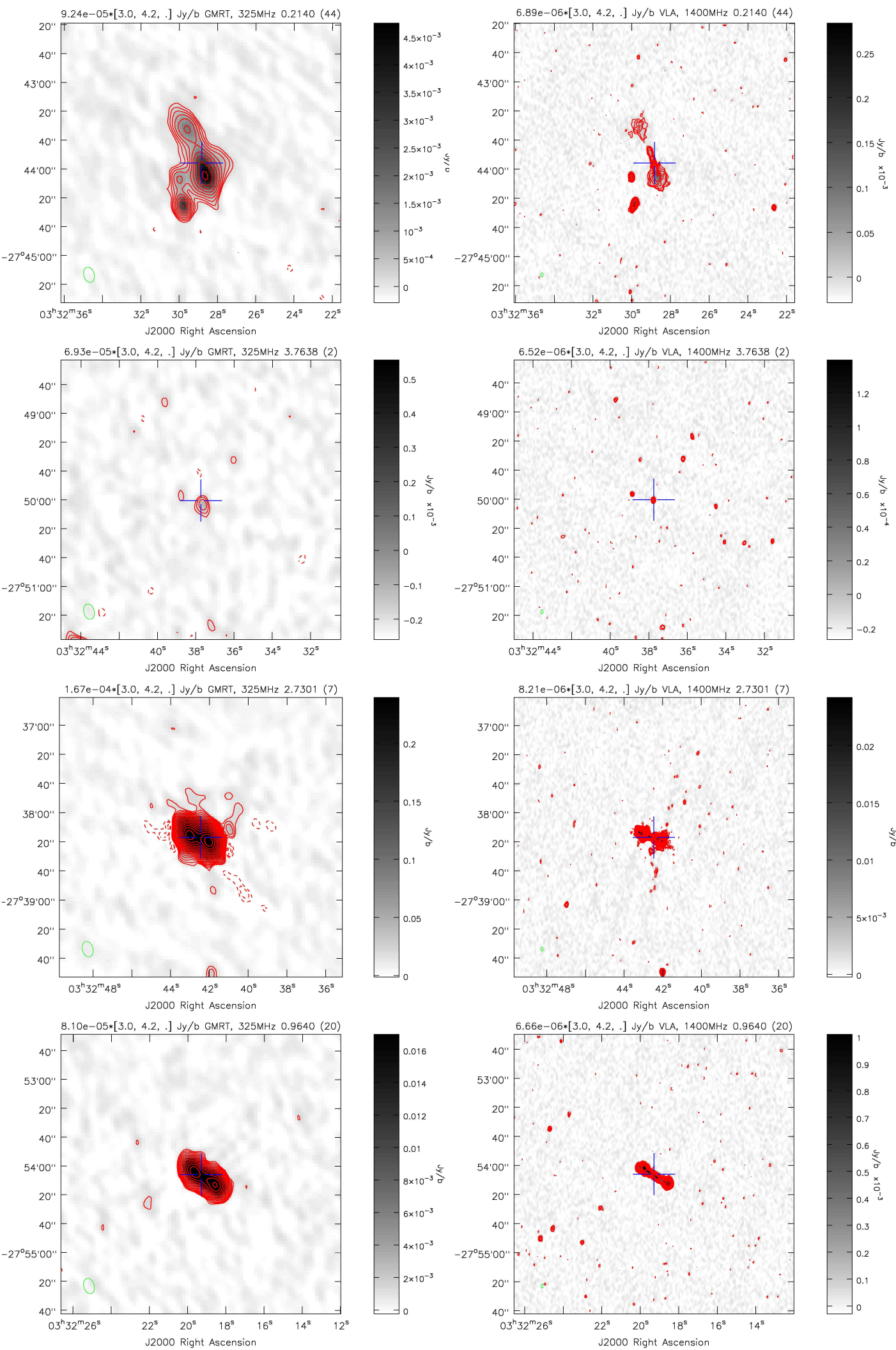

Figure 4: Our GMRT 325-MHz images are shown along with the VLA 1400-MHz images which have been plotted from the image of Miller et al. [14] for four of the sources in the ECDFS field. The redshift for each of the source is also indicated at the top right corner of the images. 

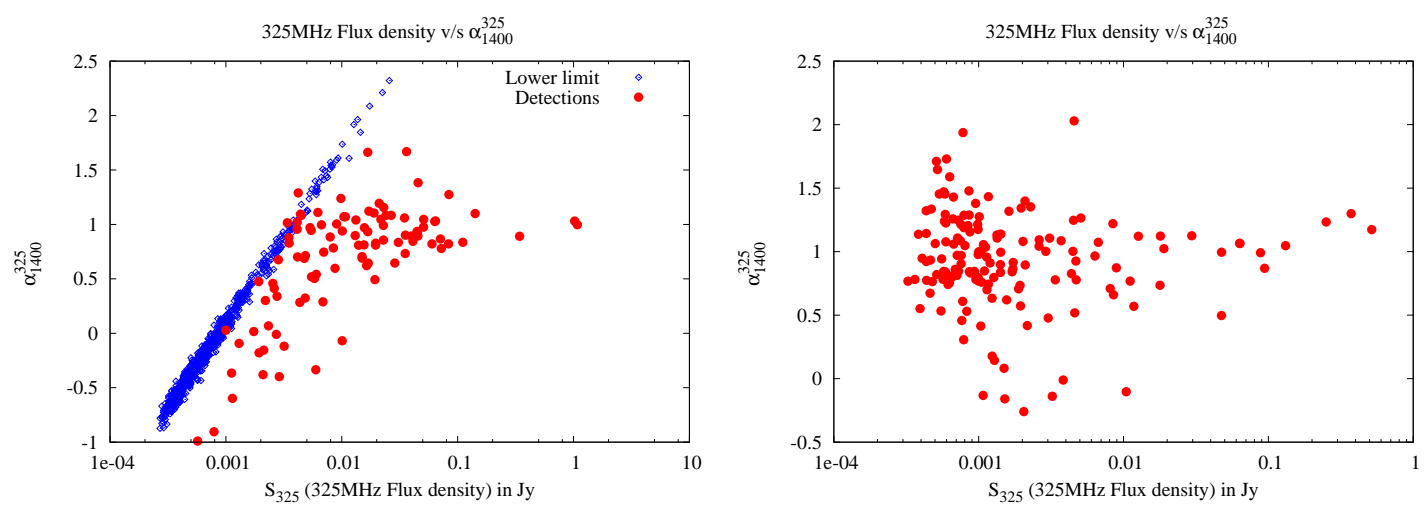

Figure 5: The spectral index vs flux density for the ELAIS-N1 (left) and ECDFS (right) sources.

source counts for the ECDFS observations, along with those obtained earlier from the ELAIS-N1 observations [12]. The source counts from the two fi elds are consistent. A comparison with the functional form fi tted by Wieringa [15] to the differential source counts ranging from about $4 \mathrm{mJy}$ to $1 \mathrm{Jy}$ from his deep 325-MHz Westerbork survey, shows clearly the flattening of the source counts at $\sim 1 \mathrm{mJy}$ at $325 \mathrm{MHz}$. A more detailed modeling of the source counts is in progress.

\section{Concluding remarks}

Deep images at low radio frequencies have helped determine their spectra over a large frequency range providing additional information to distinguish between AGN and starburst activity, These observations have shown a flattening of the source counts at low flux densities which is possibly due to a population of both starburst galaxies and low-luminosity AGN, and have helped identify many very steep-spectrum and several weak GPS sources. The very steep-spectrum sources could be high-redshift galaxies or relic radio sources and are being investigated further. It would be interesting to identify a larger sample of weak GPS sources and enquire whether their evolutionary paths are similar to those of the more luminous ones identifi ed from strong radio source surveys.

\section{References}

[1] P. Madau, L. Pozzetti and M. Dickinson, The Star Formation History of Field Galaxies, ApJ, 498, 106, (1998)

[2] A.M. Hopkins, J.F. Beacom, On the Normalization of the Cosmic Star Formation History, ApJ, 651, 142, (2006)

[3] E.B. Fomalont, K.I. Kellermann, R.B. Partridge, R.A. Windhorst, E.A. Richards, The Microjansky Sky at $8.4 \mathrm{GHz}, \mathrm{AJ}, \mathbf{1 2 3} 2402$, (2002)

[4] E.B. Fomalont, K.I. Kellermann, L.L. Cowie, P. Capak, A.J. Barger, R.B. Partridge, R.A. Windhorst, E.A. Richards, The Radio/Optical Catalog of the SSA 13 Field, ApJS, 167, 103, (2006)

[5] P. Ciliegi et al., The VVDS-VLA deep field. II. Optical and near infrared identifications of VLA S1.4 GHz>80 $\mu$ Jy sources in the VIMOS VLT deep survey VVDS-02h field, A\&A, 441, 879, (2005) 


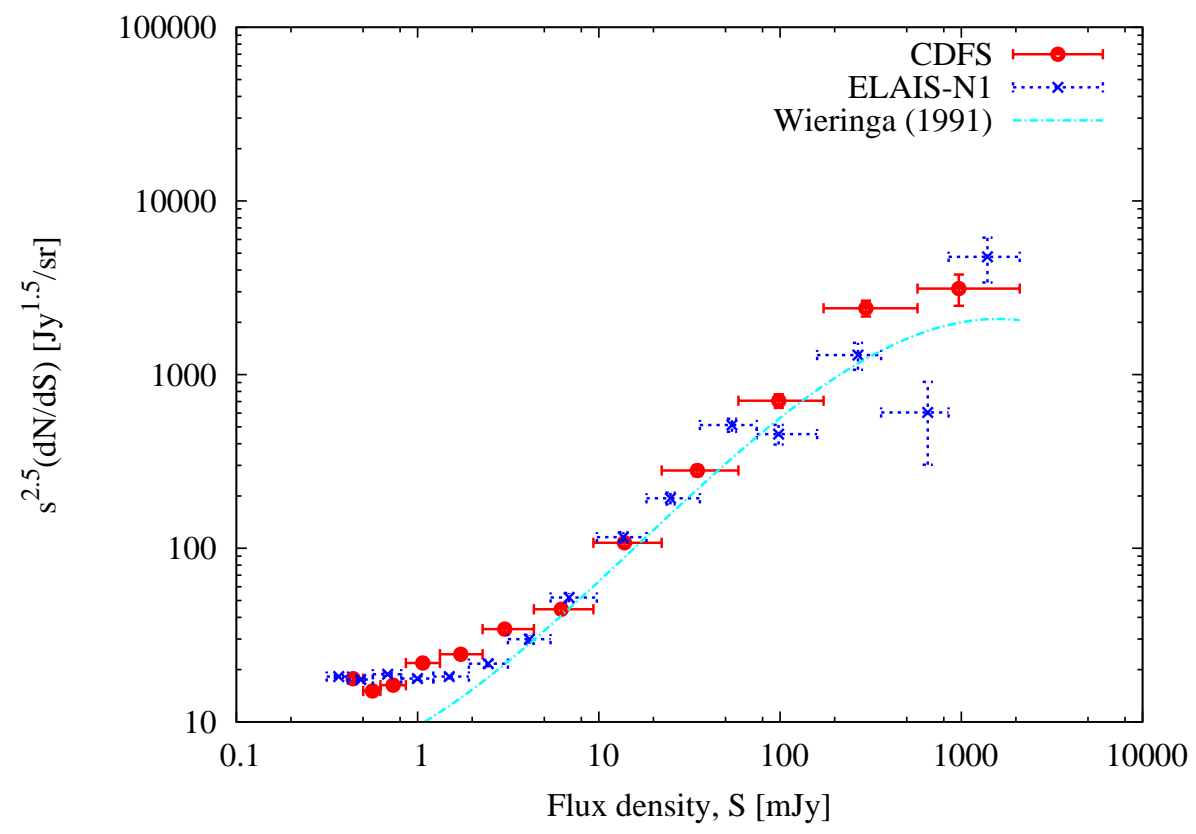

Figure 6: The normalized differential source counts at $325 \mathrm{MHz}$ for the ELAIS-N1 [12] and ECDFS fields.

[6] V. Smolcić, et al., A New Method to Separate Star-forming from AGN Galaxies at Intermediate Redshift: The Submillijansky Radio Population in the VLA-COSMOS Survey, ApJS, 177, 14, (2008)

[7] M. Y. Mao, R. Sharp, D. J. Saikia, R. P. Norris, M. Johnston-Hollitt, E. Middelberg, J. E. J. Lovell, Wide-angle tail galaxies in ATLAS, MNRAS, 406, 2578, (2010)

[8] G. Miley, C. De Breuck, Distant radio galaxies and their environments, A\&ARv, 15, 67, (2008)

[9] G. Giovannini, A. Bonafede, L. Feretti, F. Govoni, M. Murgia, F. Ferrari, G. Monti, Radio halos in nearby $(z<0.4)$ clusters of galaxies, $A \& A, \mathbf{5 0 7}, 1257,(2009)$

[10] D.J. Saikia, M. Jamrozy, Recurrent activity in Active Galactic Nuclei, BASI, 37, 63, (2009)

[11] S.K. Sirothia, D.J. Saikia, C.H. Ishwara-Chandra, N.G. Kantharia, Deep low-frequency observations with the Giant Metrewave Radio Telescope: a search for relic radio emission, MNRAS, 392, 1403, (2009)

[12] S.K. Sirothia, M. Dennefeld, D.J. Saikia, H. Dole, F. Ricquebourg, J. Roland, 325-MHz observations of the ELAIS-N1 field using the Giant Metrewave Radio Telescope, MNRAS, 395, 269, (2009)

[13] T. Garn, D.A. Green, J.M. Riley, P. Alexander, A 610-MHz survey of the ELAIS-N1 field with the Giant Metrewave Radio Telescope - observations, data analysis and source catalogue, MNRAS, 383, $75,(2008)$

[14] N. A. Miller, E.B. Fomalont, K.I. Kellermann, V. Mainieri, C. Norman, P. Padovani, P. Rosati, P. Tozzi, The VLA 1.4 GHz Survey of the Extended Chandra Deep Field-South: First Data Release, ApJS, 179, 114, (2008)

[15] M.H. Wieringa, PhD thesis, Rijksuniversiteit Leiden, (1991) 